\title{
Glucagonoma Neuroendocrine Tumor with the Clue of Necrolytic Migratory Erythematous Rash: A Case Report
}

\author{
Seyyed-Mohsen Hosseininejad ${ }^{1}$,Fateme-Sadat Hosseininejad ${ }^{2}$, Alireza Norouzi ${ }^{3}$, Masoud Khoshnia*3 \\ ${ }^{1}$ Medical Student, Student Research Committee, Golestan University of Medical Sciences, Gorgan, Iran \\ ${ }^{2}$ Environmental health engineering student, Student Research Committee, Shahrood University of Medical Sciences, Shahrood, Iran \\ ${ }^{3}$ Gastroenterologist, Golestan Research Center of Gastroenterology and Hepatology, Golestan University of Medical Sciences, Gorgan, Iran
}

Received: January 10, 2018; Published: January 22, 2018

*Corresponding author: Masoud Khoshnia, Gastroenterologist, Golestan Research Center of Gastroenterology and Herpetology, Golestan University of Medical Sciences, Gorgan, Iran, Sayyad-Shirazi Blvd, Sayyad-Shirazi Hospital, Gorgan, Golestan Province, Iran, Tel: 981732340835; Fax: 981732369210; Email: khoshniamd@gmail.com

\section{Abstract}

Background: Necrolytic migratory erythema (NME) is most commonly a paraneoplastic condition. It is the dermatologic manifestation classically associated with glucagonoma pancreatic neuroendocrine tumor. Glucagonoma syndrome has been defined by the constellation of secreting tumor associated with overproduction by the $\alpha$-cells in the pancreatic islets of Langerhans, abnormally elevated blood level of glucagon, and skin findings of NME.

Objective: Although it is not common, all physicians must have it in mind and consult for further investigation not to miss out NME.

Methods and Results: We report a case of a middle-aged female seen in clinic with longstanding skin lesions suggestive of NME revealing glucagonoma. Complete resolution of the cutaneous and systemic features occurred after surgical removal of the tumor.

Conclusion: NME is often the first clinical finding of an occult neuroendocrine pancreatic neoplasia. Physicians, especially general practitioners and dermatologists, must be aware of this condition subsequently they can be the first physician to suspect it and allow multidisciplinary management, which impacts the prognostic value. Surgical removal is the first-line therapy if early diagnosis is done before liver metastases arise.

Keywords: Glucagonoma Syndrome; Necrolytic Migratory Erythema; Paraneoplastic; Glucagonoma Syndrome; Pancreatic Neuroendocrine Tumor

\section{Introduction}

A 52-year-old Fars female presented with complaint of decrease in appetite and weight loss to the gastroenterology academic Hospital clinic as her previous original doctor .She declared a 5-year history of progressive pruritic scaly plaques as well; rashes were located on her legs, abdomen, breasts, and arms. Other sites of skin involvement including perioral/anal, groin and flexor regions were intact. She had diabetes mellitus type 2 and suffered from deep vein thrombosis of the upper limbs since 2 months ago for which she was on warfarin tablets which were discontinued 3 weeks prior to the presentation. She had already tried many treatment modalities and denied any improvement of her condition. A generalized pruritic hyperpigmented skin rashes due to possible pustular psoriasis for which she received oral glucocorticoid in her different visits by some physicians for 5 years. She was free of the rashes till two months before the admission when she discontinue corticosteroid regime and her rashes relapsed and progressed to the erythematous vesicopustular skin lesions more prominent in right upper limb, breasts and inguinal region and lower limbs while these 2 months. Her drug history included glybenclamide, azathioprine, warfarin and oral prednisolone. The family and social history were negative for any especial condition.

On admission, she had a blood pressure of $110 / 60 \mathrm{mmHg}$, pulse rate of $75 \mathrm{bpm}$, respiratory rate of 16 per minute and oral temperature of 37 . More physical examinations shown a 3+ edema of the right upper extremity and erosions and crusted plaques with erythematous and circinate borders associated with a centrifugal growth pattern leaving a copper color as it resolved (Figures 1\&2). Vesicles and pustules were also seen. She reported decrease of appetite causing a significant weight loss. Painful finger and toe paronychia with involvement of almost all her nails had negative 
repercussions on her quality of life. Skin biopsy conducted in our department demonstrated hyperkeratosis, parakeratosis, acanthosis with psoriasiform hyperplasia, spongiosis, pale-stained upper layers of the epidermis with necrotic keratinocytes, and perivascular lymphocytic inflammatory cells infiltrate. Nutritional deficiencies and paraneoplastic syndrome were suspected according to the clinical history and the progressive and unresponsive rash associated with nonspecific histopathologic findings. Blood tests revealed a normochromic normocytic anemia with hemoglobin of $107 \mathrm{~g} / \mathrm{L}$ and an elevated ferritin level at $259 \mu \mathrm{g} / \mathrm{L}$ (normal values, $31-224 \mu \mathrm{g} / \mathrm{L}$ ) with an erythrocyte sedimentation rate of: $97 \mathrm{~mm} / \mathrm{hr}$, blood glucose of $168 \mathrm{mg} / \mathrm{dl}$.

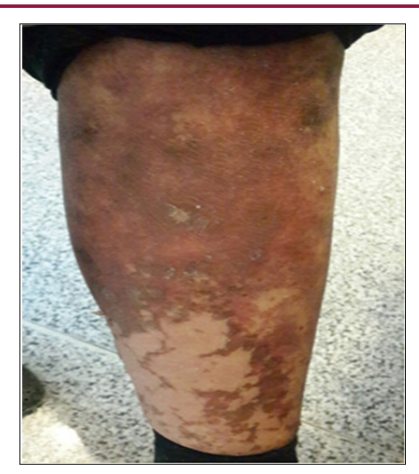

Figure 1: Vesico-pustular erythematous plaques on the leg.

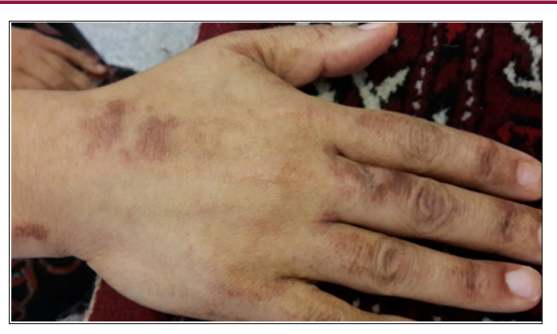

Figure 2: Hyper pigmented skin lesion on the dorsum of the hand.

Anti-transglutaminase antibodies were negative, and serum protein electrophoresis revealed a polyclonal hypergammaglobulinemia due to a reactive process associated with hyper-IgA at 5.48g/L (0.89-4.45 g/L). Hepatitis serologic tests were negative. Nutritional investigations confirmed normal levels for extended electrolytes, vitamins D and C, zinc, and vitamins B9 and B12, while niacin (B3) level was under $0.5 \mu \mathrm{g} / \mathrm{mL}(0.5-8.45 \mu \mathrm{g} / \mathrm{mL})$. Glycosylated hemoglobin (HbA1c) level was elevated at $8.3 \%$. Albumin was decreased at $24 \mathrm{~g} / \mathrm{L}(36-45 \mathrm{~g} / \mathrm{L})$ while fructosamine/protein ratio was also elevated at $4.6 \mu \mathrm{mol} / \mathrm{g}(2.9-4.0 \mu \mathrm{mol} / \mathrm{g})$. The EMG-NCV study result was in favor of polyneuropathy. Contrast-enhanced abdominal computed tomography (CT) scan revealed a $3 * 5-\mathrm{cm}$ irregular and heterogeneous calcified mass located in the body and tail of the pancreas. Gathering the gastroenterological findings, skin lesions and history of diabetes and all other data beside CT-scan findings, endocrine investigations obtained which revealed a high level of glucagon at $740 \mathrm{pg} / \mathrm{mL}(\leq 134 \mathrm{pg} / \mathrm{mL})$ and chromogranin at $565 \mathrm{ng} / \mathrm{mL}(<82 \mathrm{ng} / \mathrm{mL})$, which are neuroendocrine tumor markers associated with a normal level of insulin and C-peptide. Endoscopic ultrasound guided fine-needle aspiration biopsy confirmed neu- roendocrine cells. Imaging investigations also revealed occult left renal and right femoral vein thrombosis necessitating anticoagulation therapy with enoxaparin. Cutaneous findings were compatible with necrolytic migratory erythema (NME) related to glucagonoma pancreatic neuroendocrine tumor of the distal pancreas. Surgery to excise the mass was planned. Removal of the tumor led to a rapid resolution of the clinical features and complete normalization of glycaemia. Final histologic evaluation revealed a well-differentiated synaptophysin/chromogranin/glucagonpositive pancreatic tumor with a Ki-67 index below 2\%. There was no more invasion and Lymphadenopathy or ascites, which classified the tumor stage as pT2N0M0.

\section{Discussion}

The estimated incidence of glucagonoma syndrome is 1 in 20 million per year [1]. It is frequently diagnosed in the fifth decade of life [2]. It is a slowly developing tumor often missed initially due to nonspecific symptoms, clarifying the average diagnostic delay of more than 3 years [2]. The glucagon is the principal hormone implicated in the breakdown of glucose reserves stored in liver and muscles and also stimulates the synthesis of glucose by the liver. It has been estimated that about $90 \%$ of glucagonoma will ultimately develop diabetes mellitus [3]. It may also be responsible for the classic skin findings of NME since lowering of the glucagon blood level leads to improvement in the cutaneous disease. The increased catabolism associated with glycogenolysis may also lead to hypoaminoacidemia. Multiple nutritional deficiencies (zinc, niacin, tryptophan, proteins, amino acids, and essential fatty acids) share clinical and histological similarities with NME. Diet supplements and amino and fatty acids infusions seem to facilitate the resolution of the rash [4]. In reality, NME is not always associated with glucagonoma. Intestinal malabsorption disorders, hepatic cirrhosis, chronic pancreatitis, inflammatory bowel disease, and non-pancreatic malignancies are all considered possible causes of NME.

NME is the first clinical finding in $70 \%$ of the patients with glucagonoma syndrome [5]. The histopathological findings of epidermal necrosis explicate the classic flaccid vesicles and bullae, which rapidly become eroded, scaled, and crusted. Some plaques resolve with post-inflammatory hyperpigmentation, leaving a bronze color as new ones appear. Central clearing may give an annular appearance [5]. NME is principally located on extremities, lower abdomen, buttocks, perineum, and perioral area. 5 Nail and oral involvement is other associated manifestations. Cutaneous features can mimic bullous dermatitis; on the other hand, it may also present as psoriasiform or eczematous plaques. Differential diagnosis may include pemphigus foliaceous and IgA pemphigus, atypical bullous pemphigoid, Hailey-Hailey disease, dermatitis herpetiformis, nutritional deficiencies, necrolytic acral erythema, inverse and pustular psoriasis, contact dermatitis, nummular eczema, seborrheic dermatitis, figurate erythema, nonspecific intertrigo, and infectious diseases such as bacterial folliculitis and chronic muco-cutaneous candidiasis [1,6]. Nonspecific histopathological findings with necrotic keratinocytes in superficial 
layers of the epidermis are often described as in our case. Early histopathological changes are referred to as dyskeratotic dermatitis [6].

Skin biopsy without considering clinical picture of the disease seems to be a poor diagnostic tool for NME, which also leads to the diagnostic delays if clinical features are unrecognized enough. Classic manifestations of glucagonoma syndrome are NME, angular cheilitis, glossitis, diabetes mellitus, normochromic and normocytic anemia, venous thrombosis, weight loss, and neuropsychiatric symptoms [7]. The acronym " $4 \mathrm{D}$ " has been proposed to remember their classic characteristics, which stands for dermatosis, diabetes, deep vein thrombosis, and depression [8]. There is a significant risk of thromboembolism, which is responsible for the immediate reason of death in up to $50 \%$ of cases [6]. Our current case had multiple occult thromboses discovered by imaging studies, reinforcing the need for long-term prophylactic anticoagulation. Glucagonoma is a slowly progressive pancreatic neuroendocrine tumor and tends to metastasize later than pancreatic adenocarcinoma [9]. The main approach, surgical removal of the tumor, is considered to be the definitive treatment for glucagonoma and NME; surgical removal is the first-line choice therapy if diagnosis is made before liver metastases occur. It has been assessed than between $50 \%$ and $90 \%$ of glucagonoma cases have progressed to metastatic disease when diagnosed, which is associated with poor prognosis.

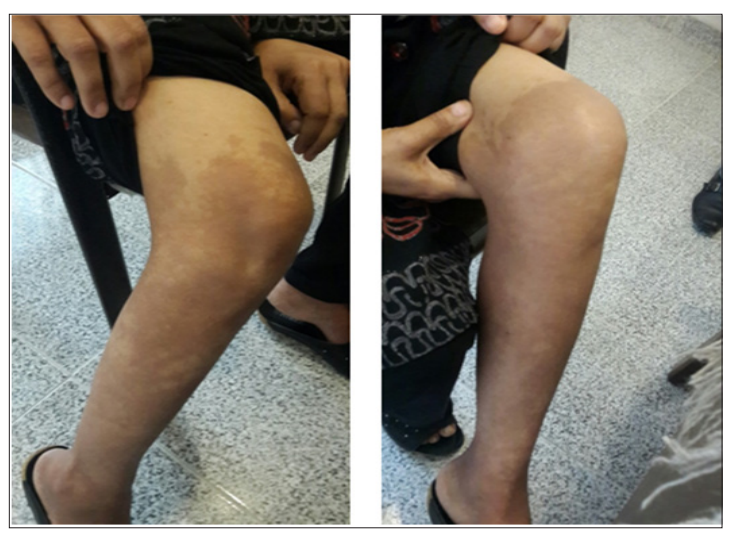

Figure 3 a\&b: resolving cutaneous inflammatory erythematous hyperpigmented skin lesions after tumor surgical removal.

Aggressive surgical therapy is associated with complete resolution of the cutaneous and systemic manifestations. If metastases have occurred, surgical planning is more complex and decisions are made on a case-by-case basis [1]. The cutaneous inflammatory lesions resolve within the first week after surgery. The mean life expectancy has been estimated between 3 and 7 years after diagnosis, and causes of death are correlated to thromboembolism, sepsis, and gastrointestinal bleeding [10]. Regarding our current case, two weeks after surgery, Skin lesions became relatively inconspicuous (Figure $3 \mathrm{a} \& \mathrm{~b}$ ) antidiabetic oral agents discontinued and she felt free of any symptoms and continue to feel healthy on 1.5 year following up visits.

\section{Conclusion}

NME is often the first clinical finding of an occult Glucagonoma. Physicians, especially general practitioners and dermatologists, should be aware of this condition subsequently they can be the first physician to suspect it and allow multidisciplinary management, which impacts the prognostic value. Surgical removal is the firstline therapy if early diagnosis is done before liver metastases arise.

\section{Conflict of interests and Funding statement}

The authors confirmed no potential conflicts of interest with respect to the research, authorship, and/or publication of this article. The authors received no financial support for the research project, authorship, and publication of this article.

\section{References}

1. John AM, Schwartz RA (2016) Glucagonoma syndrome: a review and update on treatment. J Eur Acad Dermatology Venereol 30(12): 20162022.

2. Eldor R, Glaser B, Fraenkel M, Doviner V, Salmon A, et al. (2011) Glucagonoma and the glucagonoma syndrome: cumulative experience with an elusive endocrine tumour. Clin Endocrinol (Oxf) 74(5): 593-598.

3. Wei J, Lin S, Wang C, Wu J, Qian Z, et al. (2015) Glucagonoma syndrome: a case report. Oncol Lett 10(2): 1113-1116.

4. Alexander EK, Robinson M, Staniec M, Dluhy RG (2002) Peripheral amino acid and fatty acid infusion for the treatment of necrolytic migratory erythema in the glucagonoma syndrome. Clin Endocrinol (Oxf) 57(6): 827-831.

5. Lobo I, Carvalho A, Amaral C, Machado S, Carvalho R (2010) Glucagonoma syndrome and necrolytic migratory erythema. Int J Dermatol 49(1): 2429.

6. Adam DN, Cohen PD, Sibbald RG, Ghazarian D (2003) Necrolytic migratory erythema: case report and clinical review. J Cutan Med Surg 7(4): 333-338.

7. Wermers RA, Fatourechi V, Wynne AG, Kvols LK, Lloyd RV (1996) The glucagonoma syndrome: clinical and pathologic features in 21 patients. Medicine (Baltimore) 75(2): 53-63.

8. Vinik A, Feliberti E, Perry R (2014) Glucagonoma syndrome. Endotext. South Dartmouth.

9. Kimbara S, Fujiwara Y, Toyoda M, Chayahara N, Imamura Y, et al. (2014) Rapid improvement of glucagonoma-related necrolytic migratory erythema with octreotide. Clin J Gastroenterol 7(3): 255-259.

10. Dinc B, Sahin C (2009) Metastatic glucagonoma. Eurasian J Med 41(1): 70-72. 
(C) (i) This work is licensed under Creative

Submission Link: http://biomedres.us/submit-manuscript.php

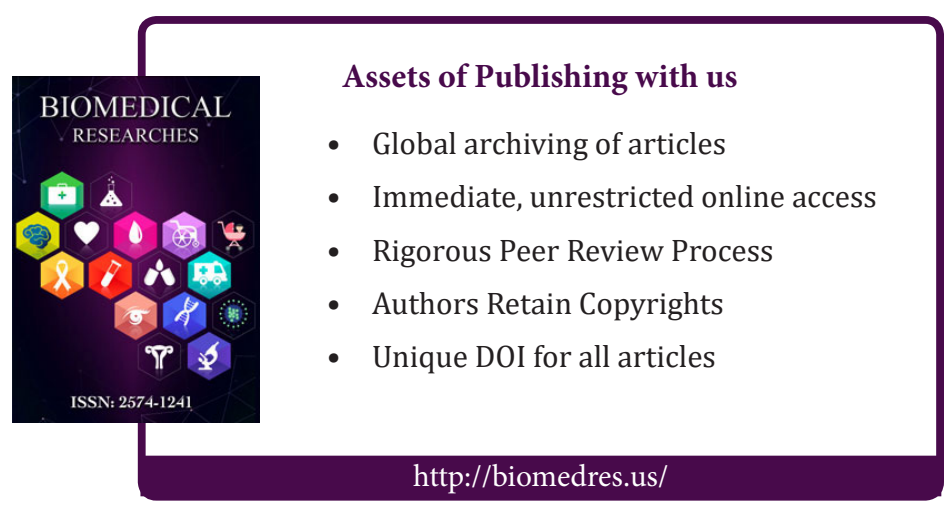

